\title{
Glutathione regulates the transfer of iron-sulfur cluster from monothiol and dithiol glutaredoxins to apo ferredoxin
}

\author{
Lei Wang ${ }^{1,2}$, Bingjie Ouyang ${ }^{1,2}$, Yifei $\mathrm{Li}^{1}$, Yingang Feng ${ }^{1}$, Jean-Pierre Jacquot ${ }^{4}$, Nicolas Rouhier ${ }^{4}$, Bin $\mathrm{Xia}^{1,2,3} \bowtie$ \\ ${ }_{1}^{1}$ Beijing Nuclear Magnetic Resonance Center, Peking University, Beijing 100871, China \\ ${ }^{2}$ College of Chemistry and Molecular Engineering, Peking University, Beijing 100871, China \\ ${ }^{3}$ School of Life Sciences, Peking University, Beijing 100871, China \\ ${ }^{4}$ UMR 1136 laM, IFR 110, Faculty of Sciences, Nancy University, Vandoeuvre 54506, France \\ $\triangle$ Correspondence: binxia@pku.edu.cn \\ Received May 24, 2012 Accepted June 7, 2012
}

\begin{abstract}
Holo glutaredoxin (Grx) is a homo-dimer that bridges a [2Fe-2S] cluster with two glutathione (GSH) ligands. In this study, both monothiol and dithiol holo Grxs are found capable of transferring their iron-sulfur (FeS) cluster to an apo ferredoxin (Fdx) through direct interaction, regardless of FeS cluster stability in holo Grxs. The ligand GSH molecules in holo Grxs are unstable and can be exchanged with free GSH, which inhibits the FeS cluster transfer from holo Grxs to apo Fdx. This phenomenon suggests a novel role of GSH in FeS cluster trafficking.
\end{abstract}

KEYWORDS iron-sulfur cluster, glutaredoxin, glutathione, ferredoxin, CD, NMR

\section{INTRODUCTION}

In the past years, several glutaredoxins (Grxs) were found to form a holo dimer bridged by a [2Fe-2S] cluster with two external glutathione (GSH) ligands (Lillig et al., 2005; Feng et al., 2006; Johansson et al., 2007, 2011; Picciocchi et al., 2007; Rouhier et al., 2007; Bandyopadhyay et al., 2008; Couturier et al., 2009; Iwema et al., 2009; Li et al., 2010; Couturier et al., 2011; Yeung et al., 2011). Five structures of holo Grxs were also determined (Lillig et al., 2005; Feng et al., 2006; Johansson et al., 2007; Rouhier et al., 2007; Iwema et al., 2009; Couturier et al., 2011; Johansson et al., 2011). Meanwhile, increasing evidence suggests that Grxs have an important function in the regulation of iron homeostasis and in iron-sulfur (FeS) cluster biogenesis (Lillig et al., 2008; Rouhier et al., 2010). Human Grx2 was found to exchange its [2Fe-2S] cluster with the human FeS cluster scaffold protein ISU (Qi and Cowan, 2011). In addition, inhibition of human Grx2 with siRNA decreases mitochondrial complex I and aconitase activities in a mammalian dopaminergic cell line (Lee et al., 2009). The yeasts cytosolic Grx3p and Grx4p can form a [2Fe-2S] cluster containing homo-dimers or Grx3/4p-Fra2p hetero-dimers with a [2Fe-2S] cluster, and function in intracellular iron sensing and trafficking ( $\mathrm{Li}$ et al., 2009; Herrero et al., 2010; Muhlenhoff et al., 2010). Yeast Grx5p was found to participate to the FeS cluster assembly in mitochondria; and in a grx 5 deletion mutant, several FeS cluster containing enzymes exhibit lower activities (Muhlenhoff et al., 2003; Alves et al., 2004a, b; Rouhier et al., 2010). Poplar GrxS14 and GrxS16, which can dimerize to assemble a [2Fe-2S] cluster, compensate for the yeast grx5 deletion mutant for $\mathrm{FeS}$ cluster assembly (Bandyopadhyay et al., 2008). Meanwhile, holo GrxS14 is capable of transferring its FeS cluster to apo ferredoxin ( $\mathrm{Fdx}$ ) under anaerobic conditions (Bandyopadhyay et al., 2008). Recently, reports state that $E$. coli GrxD can also form a [2Fe-2S] cluster containing homo-dimer or hetero-dimer with BolA, both capable of transferring the FeS cluster to apo Fdx (Yeung et al., 2011). Therefore, poplar GrxS14/S16 and E. coli GrxD, which are CGFS-type monothiol Grxs, are likely to function as FeS cluster scaffold proteins.

The [2Fe-2S] clusters on poplar GrxS14 and human Grx2 are labile, and free GSH is required to stabilize the holo proteins (Fig. S1) (Lillig et al., 2005; Berndt et al., 2007; Wang et al., 2011). On the contrary, the [2Fe-2S] cluster in poplar 
dithiol GrxC1 (YCGYC active site) is more stable (Rouhier et al., 2007), and no FeS cluster transfer activity is observed toward apo Fdx (Bandyopadhyay et al., 2008; Rouhier et al., 2010). Therefore, it is believed that the lability of FeS cluster in monothiol holo Grxs is related to their FeS cluster transfer ability (Bandyopadhyay et al., 2008; Rouhier et al., 2010). Nevertheless, the molecular mechanisms for the FeS cluster transfer reaction from holo Grxs to acceptor proteins remain unknown. In this work, we studied the mechanism of FeS cluster transfer from holo Grxs to apo Fdx using NMR and CD spectroscopies, and then analyzed the regulation of FeS cluster transfer process.

\section{RESULTS}

We probed the FeS cluster transfer reaction using poplar
GrxS14 (pGrxS14) and apo Fdx from Synechocystis by NMR spectroscopy. The NMR sample contained $0.3 \mathrm{mmol} / \mathrm{L}$ ${ }^{15} \mathrm{~N}$-labeled apo $\mathrm{Fdx}$ and $0.6 \mathrm{mmol} / \mathrm{L}$ holo pGrxS14, with 1 $\mathrm{mmol} / \mathrm{L} \mathrm{GSH}$ in PBS buffer at $\mathrm{pH}$ 7.0. A series of $2 \mathrm{D}{ }^{1} \mathrm{H}-{ }^{15} \mathrm{~N}$ HSQC spectra were recorded to monitor the cluster transfer reaction under aerobic conditions. Immediately after mixing apo $\mathrm{Fdx}$ and holo pGrxS14, six $\mathrm{NH}$ signals of apo $\mathrm{Fdx}$ were perturbed by holo pGrxS14 and their intensities were decreased (Fig. 1A, black triangles). The decrease of $\mathrm{NH}$ signal intensity was caused by the paramagnetic relaxation enhancement effect of the FeS cluster as a result of direct interaction between holo pGrxS14 and apo Fdx. As time went on, signature $\mathrm{NH}$ signals of holo $\mathrm{Fdx}$ appeared gradually with continuous increase in intensity (Fig. 2), confirming that apo Fdx receives the [2Fe-2S] cluster from holo pGrxS14 and folds into holo Fdx.

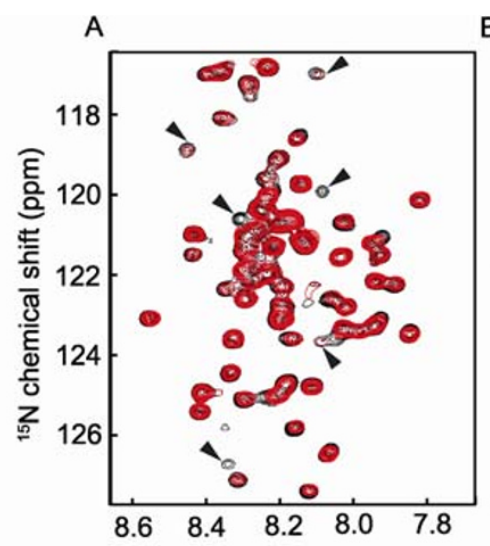

B

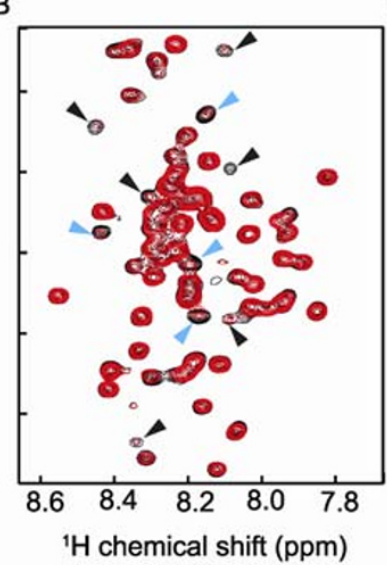

C

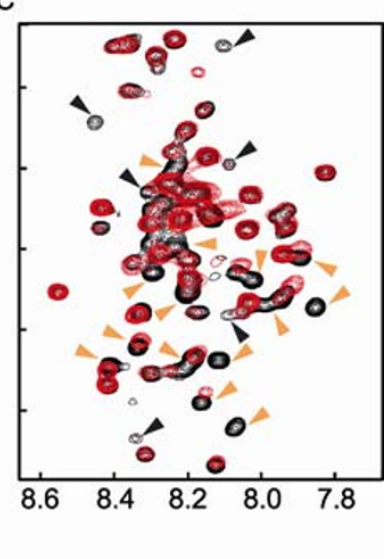

Figure 1. Interaction of apo Fdx with holo Grxs. Overlay of $2 \mathrm{D}{ }^{1} \mathrm{H}^{-15} \mathrm{~N} \mathrm{HSQC}$ spectra of apo Fdx in the absence (black) and presence (red) of holo pGrxS14 (A), holo pGrxC1 (B) or holo hGrx2 (C). The commonly perturbed signals of apo Fdx by the Grxs are indicated as black triangles. Four more $\mathrm{NH}$ signals of apo Fdx perturbed by holo pGrxC1 are indicated as cyan triangles. Different $\mathrm{NH}$ signals of apo Fdx affected by holo hGrx2 comparing to holo pGrxS14 and pGrxC1 are indicated as orange triangles.

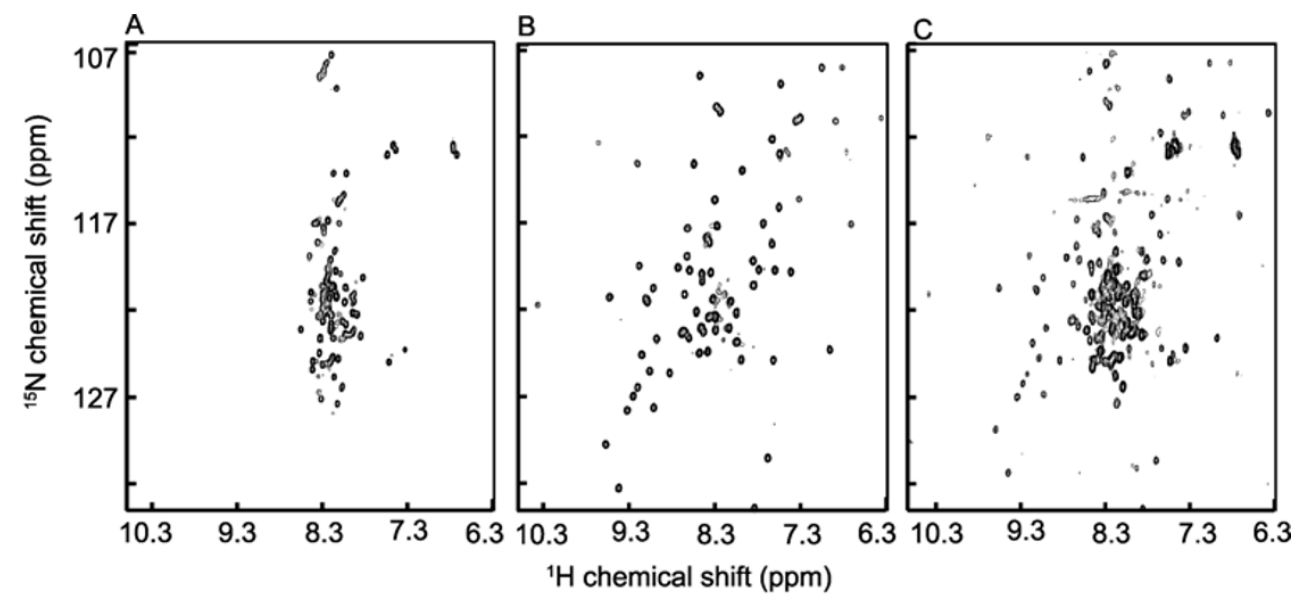

Figure 2. FeS cluster transfer assay with NMR under aerobic conditions. $2 \mathrm{D}{ }^{1} \mathrm{H}-{ }^{15} \mathrm{~N} H S Q \mathrm{C}$ spectra of apo Fdx (A), holo Fdx (B), and apo Fdx mixed with holo pGrxS14 for $1 \mathrm{~h}(\mathrm{C})$, at pH 8.0. The NMR spectra for the FeS cluster transfer from holo pGrxC1 and hGrx2 are shown in Fig. S2. 
Since it was reported that there is no sign of cluster transfer from poplar GrxC1 (pGrxC1) to apo Fdx (Bandyopadhyay et al., 2008), we investigated whether apo $\mathrm{Fdx}$ can interact with $\mathrm{pGrxC1}$ through NMR. As pGrxC1 possesses a rather stable FeS cluster (Rouhier et al., 2007; Bandyopadhyay et al., 2008), no free GSH was added in the NMR sample. All six NH signals of apo Fdx affected by holo pGrxS14 showed decreased intensities immediately after adding holo $\mathrm{pGrxC1}$, and four more $\mathrm{NH}$ signals were weakened, indicating that apo $\mathrm{Fdx}$ has additional contacts with holo pGrxC1 compared with pGrxS14 (Fig. 1B, cyan triangles). Surprisingly, we observed that after a few hours, the signature $\mathrm{NH}$ signals of holo $\mathrm{Fdx}$ appeared in $2 \mathrm{D}{ }^{1} \mathrm{H}-{ }^{15} \mathrm{~N}$ HSQC spectra (Fig. S2). This finding suggests that holo pGrxC1 can also transfer the FeS cluster to apo Fdx. Furthermore, signature $\mathrm{NH}$ signals of holo $\mathrm{Fdx}$ were observed when we repeated the cluster transfer assay in the presence of EDTA, indicating that the formation of holo Fdx was not due to non-specific FeS cluster assembly. Clearly, the NMR assay produced different results from the previous FeS cluster transfer assay using CD spectroscopy (Bandyopadhyay et al., 2008). The former assay with CD was carried out in an anaerobic environment in the presence of free GSH at $\mathrm{pH} 8.0$, whereas the present NMR assay was carried out under aerobic conditions at $\mathrm{pH} 7.0$ without free GSH. The NMR cluster transfer assay was repeated for $\mathrm{pGrxC1}$ in the presence of $20 \mathrm{mmol} / \mathrm{L} \mathrm{GSH}$. No $\mathrm{NH}$ signal of holo Fdx could be observed even after $12 \mathrm{~h}$, consistent with the results reported previously. This result suggested that free GSH has an inhibitory effect on FeS cluster transfer from holo $\mathrm{pGrxC1}$ to apo $\mathrm{Fdx}$.

NMR spectroscopy is not sensitive for monitoring the early stage of the FeS cluster transfer, so we further analyzed the FeS cluster transfer from holo Grxs to apo Fdx using CD spectroscopy under aerobic environment for different solution conditions. We first compared the rate of cluster transfer for pGrxS14 between $\mathrm{pH} 7.0$ and 8.0, and found that the initial transfer rate at $\mathrm{pH} 8.0$ is 1.4 times faster than that at $\mathrm{pH} 7.0$. Thus, the cluster transfer is faster at pH 8.0 (Fig. 3). This result is similar to the situation described for holo ISU, which also displays a $\mathrm{pH}$-dependent FeS cluster transfer rate toward apo Fdx. It was proposed that the deprotonation of the thiol group in cysteine would facilitate cluster transfer at higher $\mathrm{pH}$ values (Wu et al., 2002). We then compared the cluster transfer rates for pGrxS14 in the presence of 1 and $20 \mathrm{mmol} / \mathrm{L} \mathrm{GSH}$. Free GSH was found to have an inhibitory effect on the FeS cluster transfer from holo GrxS14 to apo $\mathrm{Fdx}$, and the initial rate is $50 \%$ slower with $20 \mathrm{mmol} / \mathrm{L}$ GSH (Fig. 3).
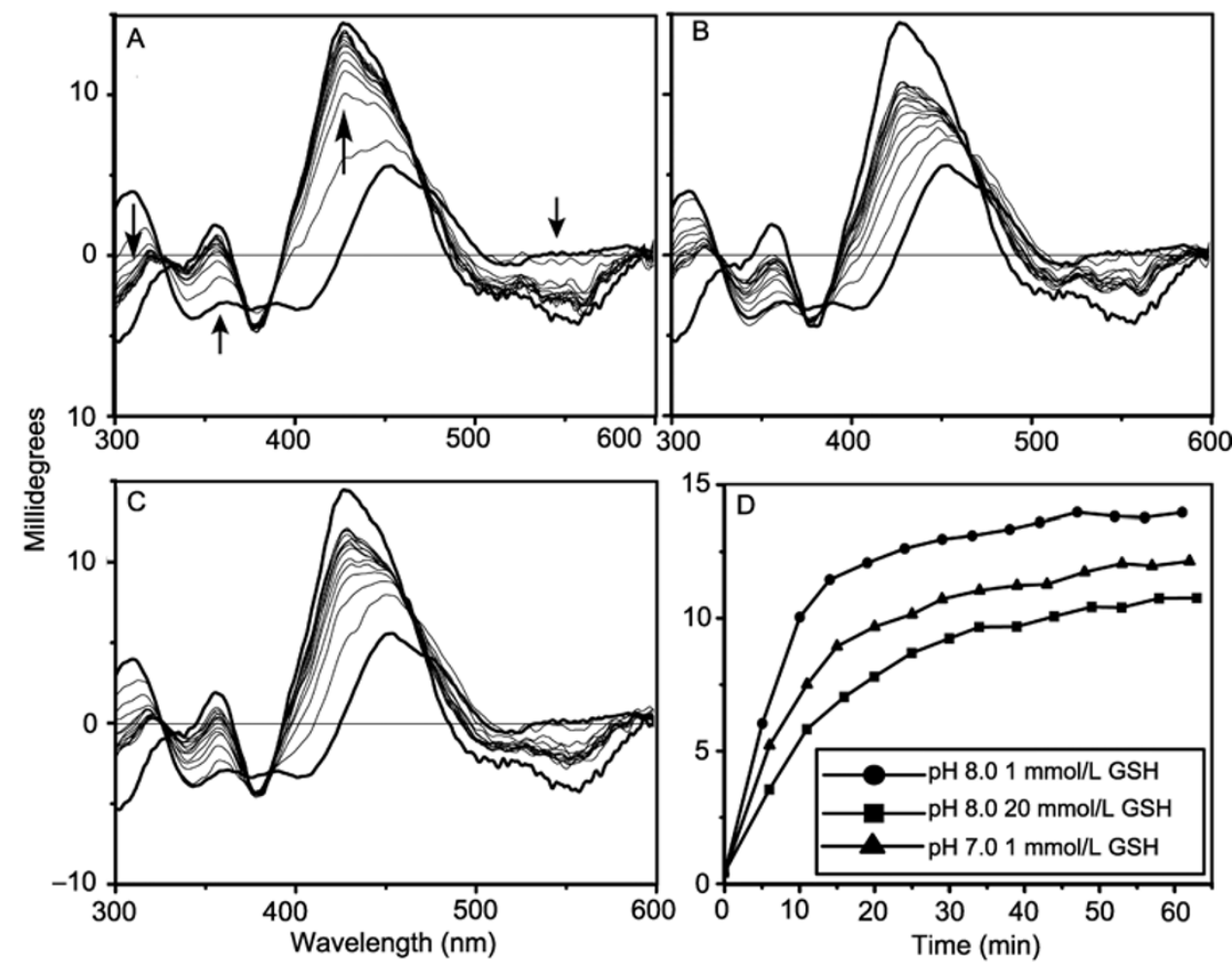

Figure 3. FeS cluster transfer assay for pGrxS14 with CD. CD spectra recorded at 5 min intervals for $1 \mathrm{~h}$ at $25^{\circ} \mathrm{C}$ (thin lines) are shown for cluster transfer assays in the presence of $1 \mathrm{mmol} / \mathrm{L} \mathrm{GSH}$ at pH $8.0(\mathrm{~A}), 20 \mathrm{mmol} / \mathrm{L} \mathrm{GSH}$ at pH 8.0 (B), or $1 \mathrm{mmol} / \mathrm{L}$ $\mathrm{GSH}$ at pH $7.0(\mathrm{C})$. The increasing CD intensity at $428 \mathrm{~nm}$ with time (D) is plotted to indicate the rate of cluster transfer. The arrows indicated the direction of intensity change with time in A. Thick lines correspond to CD spectra of holo pGrxS14 and holo Fdx. 
The assay with CD demonstrated that $45 \%$ FeS cluster was transferred from holo pGrxC1 to apo $\mathrm{Fdx}$ in $3 \mathrm{~h}$ without free $\mathrm{GSH}$, and $20 \mathrm{mmol} / \mathrm{L} \mathrm{GSH}$ almost completely abolished the cluster transfer (Fig. 4). Meanwhile, holo pGrxC1 was relatively stable, and only $3 \%$ of the cluster of pGrxC1 was degraded under aerobic conditions in $3 \mathrm{~h}$, without free $\mathrm{GSH}$ (Fig. S1). FeS cluster transfer assays with holo human Grx2 (hGrx2), another dithiol Grx with a [2Fe-2S] cluster, was performed. Findings showed that it is also capable of transferring FeS cluster to apo Fdx as monitored with either NMR or CD (Fig. S2 and Fig. 4). Unexpectedly, adding holo hGrx2 resulted in signal missing or chemical shift change for much more $\mathrm{NH}$ peaks of apo Fdx (Fig. 1C, orange triangles), which are not affected by holo pGrxS14 and pGrxC1. This finding indicates that there are much more residues in apo $\mathrm{Fdx}$ involved in the interaction with holo hGrx2, and the interaction pattern between apo $\mathrm{Fdx}$ and holo hGrx2 may be quite different from those of pGrxS14 and pGrxC1. Up to 36\% FeS cluster was transferred from holo hGrx2 to apo Fdx in $3 \mathrm{~h}$ (Fig. 4 ), whereas $13 \%$ of the cluster was degraded for holo hGrx2 in the presence of free GSH under aerobic conditions (Fig.
S1). The efficiency of cluster transfer for hGrx2 is comparable to that of holo pGrxC1, and both are lower than that of monothiol pGrxS14. Free GSH can also inhibit FeS cluster transfer by holo hGrx2, wherein the cluster transfer initial rate is reduced by $40 \%$ with $20 \mathrm{mmol} / \mathrm{L} \mathrm{GSH}$ (Fig. 4). The addition of EDTA did not affect the cluster transfer, as indicated by NMR experiments. Therefore, it is not an artifact for holo hGrx2 to transfer its FeS cluster to apo Fdx. Overall, both monothiol and dithiol Grxs possess the ability to transfer FeS cluster to apo Fdx, regardless of FeS cluster stability. The cluster transfer rate is inhibited by free GSH.

Free GSH is required to stabilize holo pGrxS14 and hGrx2 because their FeS clusters are lost very quickly during an aerobic purification without GSH (Fig. S1) (Lillig et al., 2005; Wang et al., 2011). One possible reason is that free GSH can bind holo Grxs and stabilize the complex of holo proteins. However, as 2D ${ }^{1} \mathrm{H}-{ }^{15} \mathrm{~N}$ HSQC spectra of ${ }^{15} \mathrm{~N}$-labeled holo pGrxS14 with $2 \mathrm{mmol} / \mathrm{L}$ and $20 \mathrm{mmol} / \mathrm{L}$ GSH are identical, no additional GSH binding sites are present on holo pGrxS14, which rules out direct binding between free GSH and holo pGrxS14. Another possibility is that the ligand $\mathrm{GSH}$ in holo
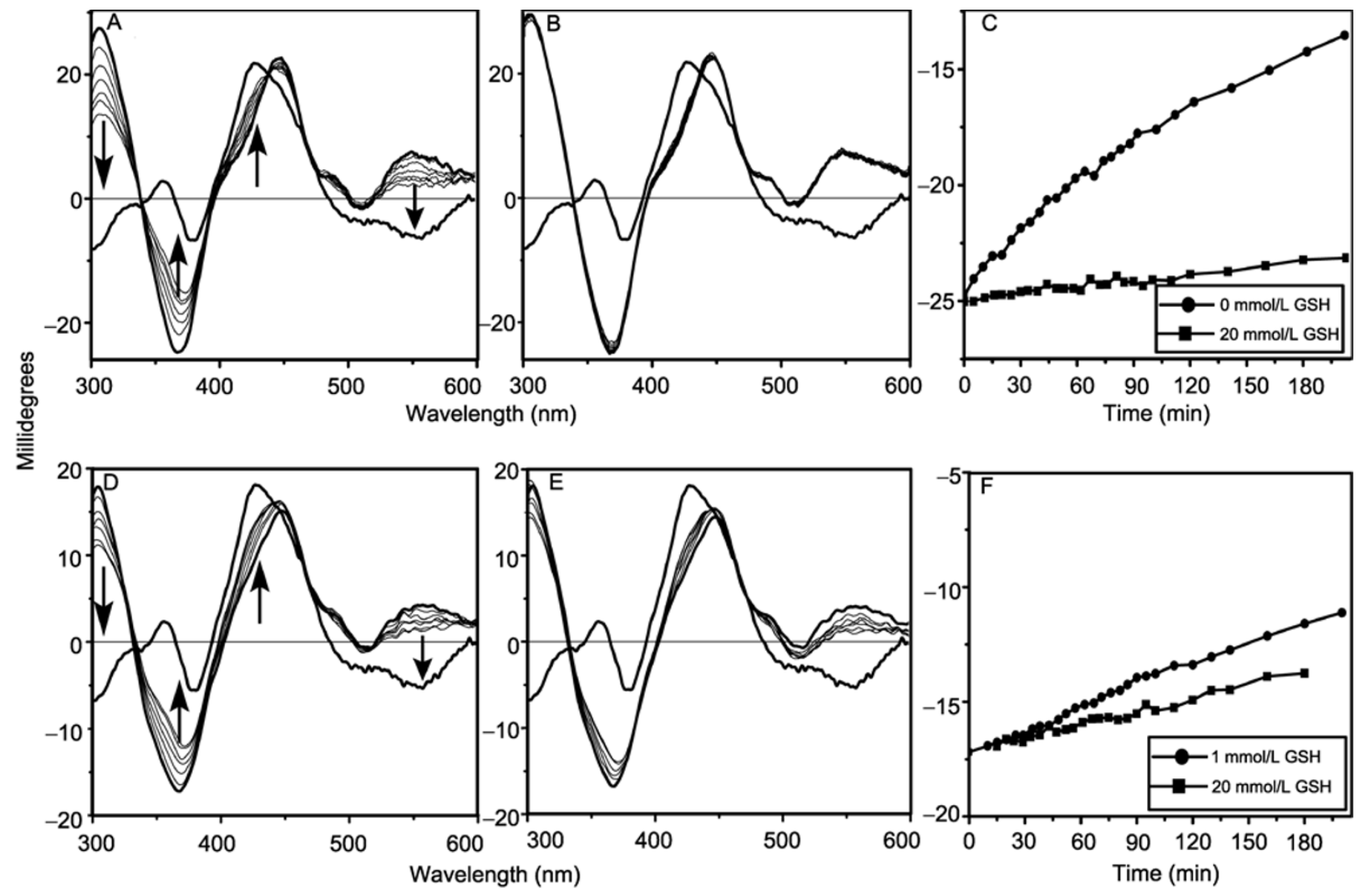

Figure 4. FeS cluster transfer assay for pGrxC1 and hGrx2 with CD. CD spectra recorded at 30 min intervals for $3 \mathrm{~h}$ at $25^{\circ} \mathrm{C}$ (thin lines) are shown for holo pGrxC1 without GSH (A) or with $20 \mathrm{mmol} / \mathrm{L} \mathrm{GSH}(\mathrm{B})$, and holo hGrx2 with $1 \mathrm{mmol} / \mathrm{L} \mathrm{GSH}$ (D) or 20 $\mathrm{mmol} / \mathrm{L} \mathrm{GSH}(\mathrm{E})$, at $\mathrm{pH}$ 8.0. The increasing CD intensities at $367 \mathrm{~nm}$ with time are plotted to indicate the rate of cluster transfer for holo pGrxC1 (C) and hGrx2 (F). The arrows indicated the direction of intensity change with time in A and D. Thick lines correspond to CD spectra of holo Grxs and holo Fdx. 
pGrxS14 falls off easily and free GSH can fill the ligand site of the FeS cluster. To test this supposition, we prepared a $0.5-\mathrm{mmol} / \mathrm{L}{ }^{15} \mathrm{~N}$-labeled holo pGrxS14 NMR sample with 2 $\mathrm{mmol} / \mathrm{L}$ unlabeled $\mathrm{GSH}$ in the buffer and added diothionite into the sample under aerobic conditions to disrupt the FeS cluster and allow the release of ligand GSH into the buffer. After removing the precipitated protein, the sample was analyzed with NMR (Fig. S3). The volume of GSH NH signal from the resulting sample was only 1.46 -fold higher than that of the buffer alone (with $2 \mathrm{mmol} / \mathrm{L} \mathrm{GSH}$ ), suggesting that the released ligand $\mathrm{GSH}(1 \mathrm{mmol} / \mathrm{L})$ is unlabeled. As the ${ }^{15} \mathrm{~N}$-labeled holo pGrxS14 was produced in $E$. coli grown in M9 medium containing ${ }^{15} \mathrm{NH}_{4} \mathrm{Cl}$, the ligand $\mathrm{GSH}$ in the holo protein produced should be fully ${ }^{15} \mathrm{~N}$-labeled. If the ${ }^{15} \mathrm{~N}$-labeled ligand $\mathrm{GSH}$ is stable, a theoretical 137-fold increase in $\mathrm{NH}$ signal volume of $\mathrm{GSH}$ after the disruption of the FeS cluster would be observed, because the ${ }^{15} \mathrm{~N}$ natural abundance of the unlabeled GSH in the buffer is only $0.366 \%$. The absence of ${ }^{15} \mathrm{~N}$-labeled $\mathrm{GSH}$ ligand indicates that the ${ }^{15} \mathrm{~N}$-labeled GSH ligand produced in E. coli was completely replaced by unlabeled $\mathrm{GSH}$ in the buffer during purification process $(\sim 5 \mathrm{~h})$. Therefore, the ligand GSH in holo pGrxS14 exchanges with free GSH in the buffer.

As the FeS cluster of holo pGrxC1 is rather stable, free GSH is absent for the purification process, and the ${ }^{15} \mathrm{~N}$-labeled holo pGrxC1 sample contains ${ }^{15} \mathrm{~N}$-labeled $\mathrm{GSH}$ (Feng et al., 2006). We also tested whether the ligand GSH in holo pGrxC1 can exchange with free GSH. We carried out 2D ${ }^{1} \mathrm{H}-{ }^{15} \mathrm{~N} \mathrm{HSQC}$ experiments to monitor the ${ }^{15} \mathrm{~N}$-labeled holo pGrxC1 $(0.5 \mathrm{mmol} / \mathrm{L})$ in the presence of $10 \mathrm{mmol} / \mathrm{L}$ unlabeled $\mathrm{GSH}$. As the ${ }^{15} \mathrm{~N}$-labeled ligand $\mathrm{GSH}$ is not visible in the NMR spectrum due to the paramagnetic effect of the FeS cluster, we only observed the natural abundant ${ }^{15} \mathrm{~N}$ signals from the $10 \mathrm{mmol} / \mathrm{L}$ unlabeled GSH in the buffer. However, the intensity and volume of $\mathrm{NH}$ signals from the ${ }^{15} \mathrm{~N}$-labeled protein did

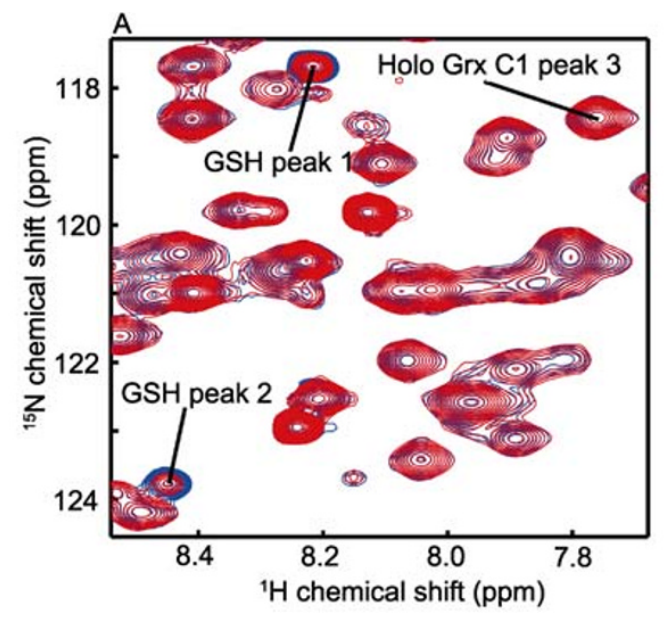

not change with time, whereas the intensity and volume of the two $\mathrm{NH}$ signals of $\mathrm{GSH}$ continuously increased, indicating that ${ }^{15} \mathrm{~N}$-labeled ligand $\mathrm{GSH}$ exchanged with the free unlabeled GSH. Thus, ${ }^{15} \mathrm{~N}$-labeled ligand $\mathrm{GSH}$ was released into the buffer and contributed to the intensity and volume increase of $\mathrm{NH}$ signals of GSH (Fig. 5). After $15 \mathrm{~h}$, the intensity of GSH signal increased 3.5-fold, indicating that $20 \%$ of ${ }^{15} \mathrm{~N}$-labeled ligand $\mathrm{GSH}$ was replaced by unlabeled free $\mathrm{GSH}$ and released into the sample buffer. Therefore, the ligand GSH in holo pGrxC1 exchanges with free GSH in the buffer, although the ligand GSH is hardly dissociated from the FeS cluster in holo pGrxC1 by itself.

\section{DISCUSSION}

Monothiol and dithiol holo Grxs are capable of transferring the FeS cluster to apo Fdx in aerobic environments through direct interaction, regardless of the stability of the FeS cluster, although the transfer of FeS cluster from poplar GrxS14 to Synechocystis Fdx is much more efficient than those from poplar GrxC1 and human Grx2. Both pGrxS14 and hGrx2 have labile FeS clusters, so the efficiency difference is not related to the FeS cluster stability of holo Grxs (Fig. S1). Our NMR data also demonstrated that apo Fdx has different interaction patterns compared with the three holo Grxs.

Although it was suggested that the ligand $\mathrm{GSH}$ of holo hGrx2 can exchange with free GSH based on the observation that incubation of unlabeled holo hGrx2 with radio-labelled GSH can result in radio-labelled holo hGrx2 (Lillig et al., 2005; Berndt et al., 2007), the data do not rule out the possibility that the holo hGrx2 is labelled by simply binding additional $\mathrm{GSH}$. We also treated the ${ }^{15} \mathrm{~N}$-labeled holo hGrx2 containing $2 \mathrm{mmol} / \mathrm{L}$ unlabeled GSH in the NMR buffer with dithionite. The ${ }^{15} \mathrm{~N}$-labeled ligand $\mathrm{GSH}$ produced in $E$. coli was completely replaced by unlabeled free GSH in the buffer during

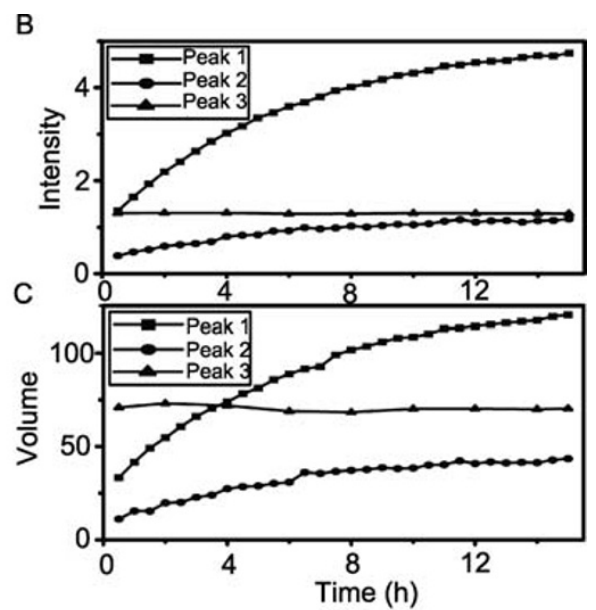

Figure 5. The exchange between the ligand GSH of holo pGrxC1 and the free GSH in buffer. Overlay of $2 \mathrm{D}{ }^{1} \mathrm{H}-{ }^{15} \mathrm{~N} \mathrm{HSQC}$ spectra of holo $\mathrm{pGrxC1}$ in the presence of $10 \mathrm{mmol} / \mathrm{L}$ unlabeled $\mathrm{GSH}$ at $0 \mathrm{~h}$ (red) and after $15 \mathrm{~h}$ (blue) (A). The change of $\mathrm{NH}$ signal intensity $(B)$ or volume $(C)$ versus time for peaks indicated in $A$. 
purification process $(\sim 5 \mathrm{~h})$, the same as that of holo pGrxS14. Therefore, in this study, we provided the first direct evidence demonstrating that ligand GSH molecules of the FeS cluster in holo pGrxS14, pGrxC,1 and hGrx2 can be replaced by free GSH in the buffer regardless of the stability of the FeS cluster. Thus, it is very likely that the ligand GSH in all holo Grxs can exchange with free GSH. These data indicate that the coordination bond between ligand GSH cysteine and the iron of the cluster is relatively weak, which can be easily substituted by other cysteines containing molecules such as apo Fdx. Therefore, we propose that to complete the FeS cluster transfer reaction process from holo Grxs to apo Fdx, cysteine residues of apo Fdx should first substitute the two GSH ligands of the [2Fe-2S] cluster in holo Grxs, followed by the two cysteine ligands of Grxs. This procedure results in a direct competition between free GSH molecules and apo Fdx, causing an inhibitory effect on the cluster transfer reaction (Fig. 6). We also suppose that the different interaction patterns of apo Fdx with the three holo Grxs are related to cluster transfer efficiency. The detailed reaction mechanism for FeS cluster transfer, e.g., how [2Fe-2S] cluster ligands are substituted, remains to be explored.

GSH is a key factor in the maintenance of the cellular redox status and an important component of the cytoplasmic labile iron pool. The level of cellular GSH can reach the millimolar concentration range (Rouhier et al., 2008; Zechmann and Russell, 2011). Inside the cell, the concentration of GSH and the GSH/GSSG ratio can vary markedly in different tissues and cell types. They also change in response to different physiological or pathological conditions, such as oxidation stress (Rouhier et al., 2008). Therefore, if Grxs serve as FeS cluster scaffold proteins in vivo, their functions are regulated by GSH. In different cellular compartments or under different physiological conditions, the efficiency of FeS cluster transfer varies. The FeS cluster transfer of holo Grxs is inhibited when the free GSH concentration is high, whereas it is accelerated when the free GSH content is low due to oxidative stress or translocation of holo Grxs.

Grxs have an important function in the regulation of iron

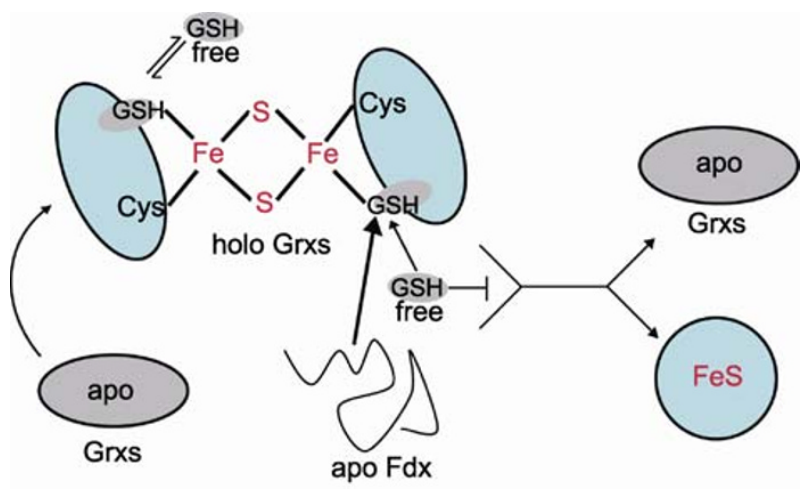

Figure 6. The proposed mechanism of FeS cluster transfer from holo Grxs to apo Fdx. homeostasis and in the FeS cluster biogenesis system (Lillig et al., 2008; Rouhier et al., 2010), recently, GSH was also found to involve in the FeS cluster biogenesis system. Defects in the biosynthesis of cellular FeS proteins are associated with increases in GSH levels (Sipos et al., 2002). In a mammalian dopaminergic cell line, depletion of GSH, which results in the defect of holo Grxs, decreases iron incorporation into mitochondrial complex I and aconitase (Lee et al., 2009). However, the exact functions of GSH and Grxs in this process remain unclear. More in vitro and in vivo studies are needed to reveal their functions.

\section{MATERIALS AND METHODS}

\section{Protein sample preparation}

The holo forms of pGrxS14, pGrxC1, hGrx2, and Fdx from Synechocystis sp. PCC 6803 were purified according to published procedures (Feng et al., 2005; Lillig et al., 2005; Feng et al., 2006; Bandyopadhyay et al., 2008; Wang et al., 2011). After the purification of holo Grxs, UV-Vis absorption spectra of holo Grxs were detected. For holo GrxS14, $A_{410 \mathrm{~nm}} / \mathrm{A}_{280 \mathrm{~nm}}$ was 0.27 . $\mathrm{A}_{430 \mathrm{~nm}} / \mathrm{A}_{280 \mathrm{~nm}}$ was 0.41 and 0.48 in the UV-Vis absorption spectra of holo pGrxC1 and hGrx2, respectively. For the purified holo $F d x$, the ratio of $A_{422 n m} / A_{280 n m}$ was 0.56 . Apo Fdx was obtained after disassembling the FeS cluster from holo $\mathrm{Fdx}$ by treating the protein with $100 \mathrm{mmol} / \mathrm{L}$ EDTA and potassium ferricyanide at room temperature under aerobic conditions for about $12 \mathrm{~h}$. Then, EDTA and potassium ferricyanide were removed by ultracentrifugation filtering with $50 \mathrm{mmol} / \mathrm{L}$ PBS and $50 \mathrm{mmol} / \mathrm{L}$ $\mathrm{NaCl}$ at $\mathrm{pH}$ 7.0. The concentration of apo Fdx protein was determined from its absorption at $A_{280 \mathrm{~nm}}$ and a theoretical extinction coefficient of $9315 \mathrm{~mol} / \mathrm{L}^{-1} \cdot \mathrm{cm}^{-1}$.

\section{FeS cluster transfer assay with NMR}

All NMR experiments were performed on a Bruker Avance $500 \mathrm{MHz}$ spectrometer or a Bruker Avance $700 \mathrm{MHz}$ spectrometer using a cryoprobe. For the FeS cluster transfer assay, 2D ${ }^{1} \mathrm{H}-{ }^{15} \mathrm{~N}$ HSQC spectrum of apo Fdx was first recorded for a $0.3-\mathrm{mmol} / \mathrm{L}{ }^{15} \mathrm{~N}$-labeled apo Fdx sample with $10 \mathrm{mmol} / \mathrm{L}$ DTT at $25^{\circ} \mathrm{C}$. Then, holo Grxs were added to the NMR sample under aerobic conditions to a $0.6-\mathrm{mmol} / \mathrm{L}$ final concentration to initiate the cluster transfer reaction, which was monitored by recording a series of $2 \mathrm{D}{ }^{1} \mathrm{H}-{ }^{15} \mathrm{~N} H S Q C$ spectra.

For the FeS cluster transfer from holo pGrxS14 or holo hGrx2 to apo Fdx, $1 \mathrm{mmol} / \mathrm{L} \mathrm{GSH}$ was included in the samples. In the case of the holo pGrxC1, the experiments were carried out either without $\mathrm{GSH}$ or in the presence of $20 \mathrm{mmol} / \mathrm{L} \mathrm{GSH}$. The NMR samples were prepared either in $50 \mathrm{mmol} / \mathrm{L}$ PBS buffer with $50 \mathrm{mmol} / \mathrm{L} \mathrm{NaCl}$ at $\mathrm{pH}$ 7.0 or in $100 \mathrm{mmol} / \mathrm{L}$ Tris- $\mathrm{HCl}$ buffer at $\mathrm{pH} 8.0$.

\section{FeS cluster transfer assay with CD}

The FeS cluster transfer assay with CD spectroscopy was similar to the previously reported procedure (Bandyopadhyay et al., 2008). The samples containing $45 \mu \mathrm{mol} / \mathrm{L}$ apo $\mathrm{Fdx}$ with $5 \mathrm{mmol} / \mathrm{L}$ DTT and the holo Grxs were added to a final concentration of $45 \mu \mathrm{mol} / \mathrm{L}$ to initiate 
the FeS cluster transfer reaction under aerobic conditions. CD spectra were recorded using a 10-mm cuvette with a 5-min interval for a period of $60 \mathrm{~min}$ for holo pGrxS14 and $180 \mathrm{~min}$ for holo pGrxC1 and hGrx2 at $25^{\circ} \mathrm{C}$. The samples were prepared in $100 \mathrm{mmol} / \mathrm{L}$ Tris- $\mathrm{HCl}$ buffer at $\mathrm{pH} 8.0$ or $50 \mathrm{mmol} / \mathrm{L}$ PBS buffer with $50 \mathrm{mmol} / \mathrm{L} \mathrm{NaCl}$ at $\mathrm{pH}$ 7.0. A MOS-450 AF/AF-CD Fast modular UV-Vis Spectrometer/Polarimeter (BioLogic, France) was used for the measurements.

The effect of free GSH was determined by performing the FeS cluster transfer reactions without and with $20 \mathrm{mmol} / \mathrm{L} \mathrm{GSH}$ for pGrxC1 or with $1 \mathrm{mmol} / \mathrm{L}$ and $20 \mathrm{mmol} / \mathrm{L} \mathrm{GSH}$ for $\mathrm{pGrxS} 14$ and hGrx2. The CD spectrum of holo Grxs gradually changed to the spectrum of holo Fdx possessing a characteristic absorption peak at $428 \mathrm{~nm}$. The efficiency of cluster transfer from holo Grxs was determined by the time course of intensity changes at $428 \mathrm{~nm}$ for holo pGrxS14 or $367 \mathrm{~nm}$ for holo pGrxC1 and hGrx2 with simulated data of $0 \%$ to $100 \%$ cluster transfer between holo Grxs and apo Fdx. For pGrxS14, the FeS cluster transfer from holo pGrxS14 to apo Fdx was $40 \%, 18 \%$, and $28 \%$, completed after $5 \mathrm{~min}$ with the following conditions: $1 \mathrm{mmol} / \mathrm{L} \mathrm{GSH}$ at $\mathrm{pH} 8.0,20 \mathrm{mmol} / \mathrm{L} \mathrm{GSH}$ at $\mathrm{pH} 8.0$, and 1 $\mathrm{mmol} / \mathrm{L} \mathrm{GSH}$ at $\mathrm{pH}$ 7.0. For $\mathrm{pGrxC1}$, the completed FeS cluster transfer from holo $\mathrm{pGrxC} 1$ to apo $\mathrm{Fdx}$ after $1 \mathrm{~h}$ was $22 \%$ without $\mathrm{GSH}$ and $1.4 \%$ with $20 \mathrm{mmol} / \mathrm{L} \mathrm{GSH}$ at $\mathrm{pH} 8.0$, whereas the completed FeS cluster transfer from holo hGrx2 to apo Fdx after $1 \mathrm{~h}$ was $13 \%$ with $1 \mathrm{mmol} / \mathrm{L} \mathrm{GSH}$ and $8 \%$ with $20 \mathrm{mmol} / \mathrm{L} \mathrm{GSH}$ at $\mathrm{pH} 8.0$.

\section{The exchange of ligand GSH with the free GSH}

For pGrxS14, a $0.5-\mathrm{mmol} / \mathrm{L}{ }^{15} \mathrm{~N}$-labeled holo pGrxS14 sample was prepared in $30 \mathrm{mmol} / \mathrm{L}$ Tris- $\mathrm{HCl}$ buffer at $\mathrm{pH} 7.5$, with $2 \mathrm{mmol} / \mathrm{L}$ unlabeled $\mathrm{GSH}$ and $5 \mathrm{mmol} / \mathrm{L} \mathrm{DTT}$. As an internal reference for comparing peak volumes, $2 \mathrm{mmol} / \mathrm{L}$ unlabeled N-Methyl acetamide was added in the NMR sample. Dithionite was added to the sample under aerobic conditions. White precipitates appeared after the brown color of the sample was bleached for a few minutes indicating that the $[2 \mathrm{Fe}-2 \mathrm{~S}]$ cluster was completely destroyed. This observation is similar to that of holo pGrxC1 (Feng et al., 2006). However, in the case of pGrxS14, not all proteins precipitated. After removing the precipitated protein, the $2 \mathrm{D}{ }^{1} \mathrm{H}-{ }^{15} \mathrm{~N}$ HSQC spectrum was measured for the sample (Fig. S3). One NH peak (8.22 ppm, $117.68 \mathrm{ppm}$ ) from GSH could be observed in the spectrum not overlapped with $\mathrm{NH}$ signals from the protein. An NH peak (7.80 ppm, $114.15 \mathrm{ppm}$ ) for $\mathrm{N}$-Methyl acetamide was observed. We compared the volumes of this NH signal of GSH in the NMR sample buffer before and after the holo pGrxS14 was treated with dithionite. The peak volume ratio was 1.46 , indicating that the released ligand $\mathrm{GSH}(1 \mathrm{mmol} / \mathrm{L})$ was unlabeled, because ${ }^{15} \mathrm{~N}$-labeled $\mathrm{GSH}$ would result in a 137 -fold volume increase.

For pGrxC1, a-0.5-mmol/L ${ }^{15} \mathrm{~N}$-labeled holo $\mathrm{pGrxC1}$ was prepared in $50 \mathrm{mmol} / \mathrm{L}$ PBS buffer at $\mathrm{pH} 6.5$ with $20 \mathrm{mmol} / \mathrm{L}$ DTT. Up to 10 $\mathrm{mmol} / \mathrm{L}$ unlabeled $\mathrm{GSH}$ was added into the NMR sample. A series of $2 \mathrm{D}{ }^{1} \mathrm{H}-{ }^{15} \mathrm{~N}$ HSQC spectra were recorded at $25^{\circ} \mathrm{C}$ over a period of $15 \mathrm{~h}$ to monitor the change of $\mathrm{NH}$ signals. The intensities of $\mathrm{NH}$ signals from the protein did not change, but the intensities of the two $\mathrm{NH}$ signal from free GSH increased continuously, suggesting that ${ }^{15} \mathrm{~N}$-labeled $\mathrm{GSH}$ was released into the sample buffer. No signature $\mathrm{NH}$ signals for apo pGrxC1 appeared, so the increase of GSH signal intensity is not due to disassembly of holo pGrxC1. Instead, it resulted from the exchange between ligand $\mathrm{GSH}$ of holo $\mathrm{pGrxC1}$ and free $\mathrm{GSH}$.

\section{ACKNOWLEDGMENTS}

All NMR experiments were carried out at the Beijing NMR Center. We thank Cheng Zhu, Wei Zhu, and Prof. Luhua Lai for their help in the CD spectroscopy. This work was supported by grants from Ministry of Science and Technology of China (Nos. 2012CB910703 and 2006DFA31210) and National Natural Science Foundation of China (Grant No. 30570353) to BX, and the ANR Grant (No. 2010BLAN1616) to NR and JPJ.

\section{ABBREVIATIONS}

CD, circular dichroism; DTT, dithiothreitol; Fdx, ferredoxin; FeS, iron-sulfur; Grx, glutaredoxin; GSH, glutathione

\section{REFERENCES}

Alves, R., Herrero, E., and Sorribas, A. (2004a). Predictive reconstruction of the mitochondrial iron-sulfur cluster assembly metabolism. II. Role of glutaredoxin Grx5. Proteins 57, 481-492.

Alves, R., Herrero, E., and Sorribas, A. (2004b). Predictive reconstruction of the mitochondrial iron-sulfur cluster assembly metabolism: I. The role of the protein pair ferredoxin-ferredoxin reductase (Yah1-Arh1). Proteins 56, 354-366.

Bandyopadhyay, S., Gama, F., Molina-Navarro, M.M., Gualberto, J.M., Claxton, R., Naik, S.G., Huynh, B.H., Herrero, E., Jacquot, J.P., Johnson, M.K., et al. (2008). Chloroplast monothiol glutaredoxins as scaffold proteins for the assembly and delivery of [2Fe-2S] clusters. EMBO J 27, 1122-1133.

Berndt, C., Hudemann, C., Hanschmann, E.M., Axelsson, R., Holmgren, A., and Lillig, C.H. (2007). How does iron-sulfur cluster coordination regulate the activity of human glutaredoxin 2? Antioxid Redox Signal 9, 151-157.

Couturier, J., Koh, C.S., Zaffagnini, M., Winger, A.M., Gualberto, J.M., Corbier, C., Decottignies, P., Jacquot, J.P., Lemaire, S.D., Didierjean, C., et al. (2009). Structure-function relationship of the chloroplastic glutaredoxin S12 with an atypical WCSYS active site. J Biol Chem 284, 9299-9310.

Couturier, J., Stroher, E., Albetel, A.N., Roret, T., Muthuramalingam, M., Tarrago, L., Seidel, T., Tsan, P., Jacquot, J.P., Johnson, M.K., et al. (2011). Arabidopsis chloroplastic glutaredoxin C5 as a model to explore molecular determinants for iron-sulfur cluster binding into glutaredoxins. J Biol Chem 286, 27515-27527.

Feng, Y., Rouhier, N., Jacquot, J.P., and Xia, B. (2005). 1H, 15N, and 13C resonance assignments of reduced glutaredoxin $C 1$ from Populus tremula $x$ tremuloides. J Biomol NMR 31, 263-264.

Feng, Y., Zhong, N., Rouhier, N., Hase, T., Kusunoki, M., Jacquot, J.P., Jin, C., and Xia, B. (2006). Structural insight into poplar glutaredoxin $\mathrm{C} 1$ with a bridging iron-sulfur cluster at the active site. Biochemistry 45, 7998-8008.

Herrero, E., Belli, G., and Casa, C. (2010). Structural and functional diversity of glutaredoxins in yeast. Curr Protein Pept Sci 11, 659-668.

Iwema, T., Picciocchi, A., Traore, D.A., Ferrer, J.L., Chauvat, F., and 
Jacquamet, L. (2009). Structural basis for delivery of the intact [Fe2S2] cluster by monothiol glutaredoxin. Biochemistry 48, 6041-6043.

Johansson, C., Kavanagh, K.L., Gileadi, O., and Oppermann, U. (2007). Reversible sequestration of active site cysteines in a 2Fe-2S-bridged dimer provides a mechanism for glutaredoxin 2 regulation in human mitochondria. J Biol Chem 282, 3077-3082.

Johansson, C., Roos, A.K., Montano, S.J., Sengupta, R., Filippakopoulos, P., Guo, K., von Delft, F., Holmgren, A., Oppermann, U., and Kavanagh, K.L. (2011). The crystal structure of human GLRX5: iron-sulfur cluster co-ordination, tetrameric assembly and monomer activity. Biochem J 433, 303-311.

Lee, D.W., Kaur, D., Chinta, S.J., Rajagopalan, S., and Andersen, J.K. (2009). A disruption in iron-sulfur center biogenesis via inhibition of mitochondrial dithiol glutaredoxin 2 may contribute to mitochondrial and cellular iron dysregulation in mammalian glutathione-depleted dopaminergic cells: implications for Parkinson's disease. Antioxid Redox Signal 11, 2083-2094.

Li, H., Mapolelo, D.T., Dingra, N.N., Naik, S.G., Lees, N.S., Hoffman, B.M., Riggs-Gelasco, P.J., Huynh, B.H., Johnson, M.K., and Outten, C.E. (2009). The yeast iron regulatory proteins $\mathrm{Grx3} / 4$ and Fra2 form heterodimeric complexes containing a [2Fe-2S] cluster with cysteinyl and histidyl ligation. Biochemistry 48, 9569-9581.

Li, L., Cheng, N., Hirschi, K.D., and Wang, X. (2010). Structure of Arabidopsis chloroplastic monothiol glutaredoxin AtGRXcp. Acta Crystallogr D Biol Crystallogr 66, 725-732.

Lillig, C.H., Berndt, C., and Holmgren, A. (2008). Glutaredoxin systems. Biochim Biophys Acta 1780, 1304-1317.

Lillig, C.H., Berndt, C., Vergnolle, O., Lonn, M.E., Hudemann, C., Bill, E., and Holmgren, A. (2005). Characterization of human glutaredoxin 2 as iron-sulfur protein: a possible role as redox sensor. Proc Natl Acad Sci U S A 102, 8168-8173.

Muhlenhoff, U., Gerber, J., Richhardt, N., and Lill, R. (2003). Components involved in assembly and dislocation of iron-sulfur clusters on the scaffold protein Isu1p. EMBO J 22, 4815-4825.

Muhlenhoff, U., Molik, S., Godoy, J.R., Uzarska, M.A., Richter, N., Seubert, A., Zhang, Y., Stubbe, J., Pierrel, F., Herrero, E., et al. (2010). Cytosolic monothiol glutaredoxins function in intracellular iron sensing and trafficking via their bound iron-sulfur cluster. Cell Metab 12, 373-385.

Picciocchi, A., Saguez, C., Boussac, A., Cassier-Chauvat, C., and Chauvat, F. (2007). CGFS-type monothiol glutaredoxins from the cyanobacterium Synechocystis PCC6803 and other evolutionary distant model organisms possess a glutathione-ligated [2Fe-2S] cluster. Biochemistry 46, 15018-15026.

Qi, W., and Cowan, J.A. (2011). Mechanism of glutaredoxin-ISU [2Fe-2S] cluster exchange. Chem Commun (Camb) 47, 4989-4991.

Rouhier, N., Couturier, J., Johnson, M.K., and Jacquot, J.P. (2010). Glutaredoxins: roles in iron homeostasis. Trends Biochem Sci 35, 43-52.

Rouhier, N., Lemaire, S.D., and Jacquot, J.P. (2008). The role of glutathione in photosynthetic organisms: emerging functions for glutaredoxins and glutathionylation. Annu Rev Plant Biol 59, 143-166.

Rouhier, N., Unno, H., Bandyopadhyay, S., Masip, L., Kim, S.K., Hirasawa, M., Gualberto, J.M., Lattard, V., Kusunoki, M., Knaff, D.B., et al. (2007). Functional, structural, and spectroscopic characterization of a glutathione-ligated [2Fe-2S] cluster in poplar glutaredoxin C1. Proc Natl Acad Sci U S A 104, 7379-7384.

Sipos, K., Lange, H., Fekete, Z., Ullmann, P., Lill, R., and Kispal, G. (2002). Maturation of cytosolic iron-sulfur proteins requires glutathione. J Biol Chem 277, 26944-26949.

Wang, L., Ren, X., Li, Y., Rouhier, N., Jacquot, J.P., Jin, C., and Xia, B. (2011). $1 \mathrm{H}, 13 \mathrm{C}$, and $15 \mathrm{~N}$ resonance assignments of reduced GrxS14 from Populus tremula $x$ tremuloides. Biomol NMR Assign 5, 121-124.

Wu, S.P., Wu, G., Surerus, K.K., and Cowan, J.A. (2002). Iron-sulfur cluster biosynthesis. Kinetic analysis of [2Fe-2S] cluster transfer from holo ISU to apo Fd: role of redox chemistry and a conserved aspartate. Biochemistry 41, 8876-8885.

Yeung, N., Gold, B., Liu, N.L., Prathapam, R., Sterling, H.J., Willams, E.R., and Butland, G. (2011). The E. coli monothiol glutaredoxin GrxD forms homodimeric and heterodimeric FeS cluster containing complexes. Biochemistry 50, 8957-8969.

Zechmann, B., and Russell, S.D. (2011). Subcellular distribution of glutathione in the gametophyte. Plant Signal Behav 6, 1259-1262. 